\title{
Acute encephalopathy in a person with spinal cord injury and chronic renal failure, treated with high doses of thiamine
}

\author{
Stampacchia $G^{*}$, Gerini A and Gattai R \\ Spinal Cord Unit, Pisa University Hospital, Pisa, Italy
}

\begin{abstract}
Encephalopathy due to thiamine deficit is described mainly in alcohol addicted persons. Thiamine is one of the micronutrients that resulted deficient in patients with spinal cord injury (SCI), but no cases of acute encephalopathy due to thiamine deficit in SCI person is published. In this paper is reported an acute loss of consciousness in a person affected by sub-acute spinal cord injury (a spinal cord compression due to an hematoma), chronic renal failure (secondary to a nephrectomy for a widespread urinary bladder neoplasia and an atrophic contralateral kidney) in treatment by dialysis, and negative anamnesis for alcoholic abuse, recovered after four days of high doses of thiamine parenteral administration .
\end{abstract}

\begin{abstract}
Abbreviations: CT: computed tomography, EEG: electroencephalogram, MR: magnetic resonance, SCI: spinal cord injury, TDP: thiamine diphosphate, TK: transketolase
\end{abstract}

\section{Introduction}

Encephalic sufferance due to thiamine deficit is described in situation were an increased necessity of vitamin B1 happen. One of the most frequent cause is an excessive alcoholic intake. Chronic alcohol ingestion can produce a decrease in absorption of thiamine from the gastrointestinal tract and impaired utilization of thiamine in the cells. Thiamine diphosphate (TDP), the enzyme cofactor form of the vitamin, is synthesized from thiamine by the action of thiamine pyrophosphokinase, an enzyme which is directly inhibited by ethanol both in vitro and in vivo [1,2]. Three major enzymes are TDP-dependent, namely the pyruvate dehydrogenase complex, a-ketoglutarate dehydrogenase and transketolase (TK). The essential nutrient thiamine is a helper molecule required by these three enzymes involved in two pathways of carbohydrate metabolism [3]. Activities of all three TDP-dependent enzymes are reduced in autopsied cerebellar tissue from alcoholic patients who died with neuropathologically confirmed Wernicke encephalopathy [4]. The products of these pathways are needed for the generation of other essential molecules involved in brain functioning. A reduction in thiamine can interfere in numerous cellular functions that lead to serious brain disorders. Thiamine deficiency is thought to play a key role in Wernicke-Korsakoff syndrome, found predominantly in alcoholics $[4,5]$.

In patients with renal failure, encephalopathy is a common problem that may be caused by uremia, thiamine deficiency, dialysis, transplant rejection, hypertension, fluid and electrolyte's disorder or drug toxicity. Most encephalopathies are reversible, so making prompt recognition and treatment is fundamental. After hemodialysis, significant improvement of uremic encephalopathy occurs, but the level of azotemia correlates poorly with the degree of neurological dysfunction. Renal failure results in accumulation of numerous organic substances that possibly act as uremic neurotoxins, but no single metabolite has been identified as the sole cause of the clinical conditions correlated with uremia. Symptoms are usually alleviated by dialysis or successful renal transplantation. Accumulation of urea, guanidino compounds, uric acid, hippuric acid, various amino acids, polypeptides, polyamines, phenols and conjugates of phenol, phenolic and indolic acids, acetone, glucuronic acid, carnitine, myoinositol, sulphates and phosphates has been reported in the literature [6].

TK enzyme is a thiamine-dependent enzyme of the pentose phosphate pathway that is found predominantly in the myelinated structures of the nervous system and has been reported to have a critical role in the maintenance of axon-cylinder myelin sheaths [7]. This enzyme was shown to be significantly inhibited by plasma, cerebrospinal fluid and low molecular weight $(<500$ dalton) dialysate fractions obtained from patients with uremic neuropathy, but not by samples from normal subjects [8] or with high level of uremia [9]. It is also of interest that in uremic subjects, TK activity of erythrocytes was found to be abnormal but it improves following dialytic therapy [6].

\section{Case report}

A 68 -years-old man had been suffering by chronic renal failure for five months, secondary to a nephrectomy due to a widespread urinary bladder carcinoma. The patient underwent in regular dialysis treatment, three times a week, obtaining a good balance of laboratory blood exams except for hemoglobin ( $8.7 \mathrm{gr} / \mathrm{dl})$ and folic acid (2.9 ng/

Correspondence to: Stampacchia G, Spinal Cord Unit, Pisa University Hospital, Pisa, Italy, E-mail: g.stampacchia@ao-pisa.toscana.it

Key words: acute encephalopathy, spinal cord injury, thiamine deficit, transketolase, Wernicke-Korsakoff encephalopathy

Received: March 01, 2018; Accepted: March 16, 2018; Published: March 19, 2018 
$\mathrm{ml})$. Erythropoietin and blood transfusions for treating the uremic anemia and oral folic acid were administered.

One month after the surgery, he became paraplegic: a spontaneous subdural hematoma at level T8- L3 resulted to spinal Magnetic Resonance (MR) tomography. Although the surgical decompression by bilateral laminectomy from T10 to L1 and consequent evacuation of the hematoma, the resulting clinical presentation was a complete spinal cord injury, AIS A, with T12 neurological level [10]. The patient didn't present impairment of cognitive functions. He was admitted to the Spinal Unit to follow a rehabilitation program with the objective to reach enough autonomy for coming back home.

The patient had good health before the diagnosis of bladder carcinoma. He was engaged in work activity: he was a bus driver, so he could drive for many hours or many days when engaged in long travels. He followed a correct alimentation and he was not use to drink alcoholics, as his work didn't permit it.

The rehabilitation project included: prevention of bedsores and deep vein thrombosis, increasing muscular tropisms (Functional Electrical Stimulation Cycling), maintaining joint excursions (passive physiokinetic therapy), increasing trunk control (active exercises), training of transferring from bed to wheelchair and utilization of wheelchair for independent mobility. He spent 10 weeks for his rehabilitation and the several co-morbidities and the necessity of dialysis contribute to prolong the time as inpatient.

When the patient was almost ready to be discharged by the Spinal Unit, having finished his rehabilitation program, he had an acute encephalopathy. The patient was agitated, unusually angry versus nursing and medical staff, said strange thinks and was unable to reply appropriately to simple questions. During the morning he vomited once and rapidly became disoriented in space and time, developing a soporous state. He started to sleep, but he could be waked up if someone called him, staying wake only few minutes. At the neurological examination flaccid paraplegia was present, but it wasn't a new condition; he was able to move the head and the upper limbs; during Mingazzini position he showed involuntary movements like flapping tremor, especially in the right hand. A mild asymmetry in the mouth with a little fall in the right side was detected and a progressive difficulty in speaking appeared. The patient was submitted to a cranialCT and any encephalic hemorrhagic foci resulted with this imaging exam. A metabolic encephalopathy due to the chronic renal failure was excluded by the nephrologist consultant and the day before the patient was submitted to a dialysis session; the resulting blood exams were substantially good for a chronic renal failure. But in the afternoon the patient underwent to a coma state with the necessity of oxygen-therapy to restore adequate oxygen blood saturation. The eye light reflex was present, but the vision was fixed and poor conjugated ocular-motion was observed.

The day after, as the patient was still in coma with sporadic break in the spontaneous ventilation, a liquor puncture was done and both chemo-physical and infective (regarding Herpes Human Virus 1- 2, Varicella-Zoster Virus, Enterovirus and Citomegalovirus) liquor exams were negative, so an infectious encephalopathy was excluded. Electroencephalogram (EEG) showed electric anomalies on frontaltemporal-central regions of right side with contra-lateral diffusion in a diffuse slowing tracing, not responsive to eye opening (Figure 1A and 1B).

The blood ammonia value $(25 \mathrm{mcg} / \mathrm{dl})$ resulted in the normal range, then portosystemic encephalopathy was excluded. So it was hypothesized that a deficit of thiamine could be the cause of the acute encephalopathy and high doses of the vitamin were injected: $200 \mathrm{mg}$ three times a day. Simultaneously a cranial MR tomography was evaluated, but any typical lesions of Wernicke encephalopathy were observed, like cortical thinning, sulcal widening, and ventricular enlargement (all atrophic signs which might reflect rapid tissue degeneration) or mammillary body shrinkage [11-13]. There were only limited areas of abnormal signal in the peri-ventricular, sub cortical and pontine white matter, probably due to chronic vascular disease. After four days of high doses of thiamin administration, the patient recovered suddenly his normal state of conscience. After two weeks the EEG returned normal with clear responsive to open eye (Figure 2).

The patient was discharged from Spinal Unit after a week and came back home with his wheelchair. Actually he is followed as outpatient every six months and at last visit he was in stable clinical condition, continuing dialysis three times a week and maintaining, at home, passive physiokinetic therapy with cycle-ergometer.

\section{Discussion}

After an acute spinal cord lesion and consequent surgery the metabolism shift to a prevalence of catabolic processes, with a dramatic increase in energy expenditure, [14]; for this reason, in the acute post-lesion phase, the spinal cord injured persons need a specific diet with increase of calories, proteins and vitamins to compensate the increased metabolism and prevent the weight loosing [1].

Probably even the amount of thiamine utilized increases in this situation but any case report of encephalopathy due to thiamin deficit has been published. In a Canadian study the authors compare the usual dietary intakes with the nationally accepted dietary reference intakes in a representative sample of community-dwelling individuals with chronic SCI; the most important findings were that the analyzed cohort consumed a diet apparently adequate in energy and macronutrients, but inadequate in several micronutrients, including thiamine [15].

The reported case is a person with a spinal cord lesion and chronic renal failure which develops a state of coma. After having excluded metabolic encephalopathy (uremic and portosystemic), it has been hypothesized thiamine deficit. In fact end stage renal disease is a condition characterized by the presence of many unknown neurotoxins that among the many pathologic effects induce a significant reduction of TK activity with a consequent increased concentration of oxythiamine, a well-known thiamine antagonist and inhibitor of TK [16]. The sub acute SCI is a condition that require an increase intake of calories and vitamin, with a possible consequent deficit [1].

The hypothesized encephalopathy due to thiamine deficit was confirmed by the response to therapy but it wasn't supported by MR imaging which didn't show pathognomonic signals of Wernicke syndrome, probably because the simultaneous presence of SCI and chronic renal failure accelerate the process to provoke acute encephalopathy not leaving enough time to develop typical encephalic lesions.

\section{Conclusion}

A spinal cord lesion increases the metabolic need of the organism because of an increased catabolic situation. Thiamine is necessary to maintain the encephalic function; in case of an acute encephalopathy of unknown genesis in SCI person, high doses of thiamin could let a recovering of the encephalic functions. 


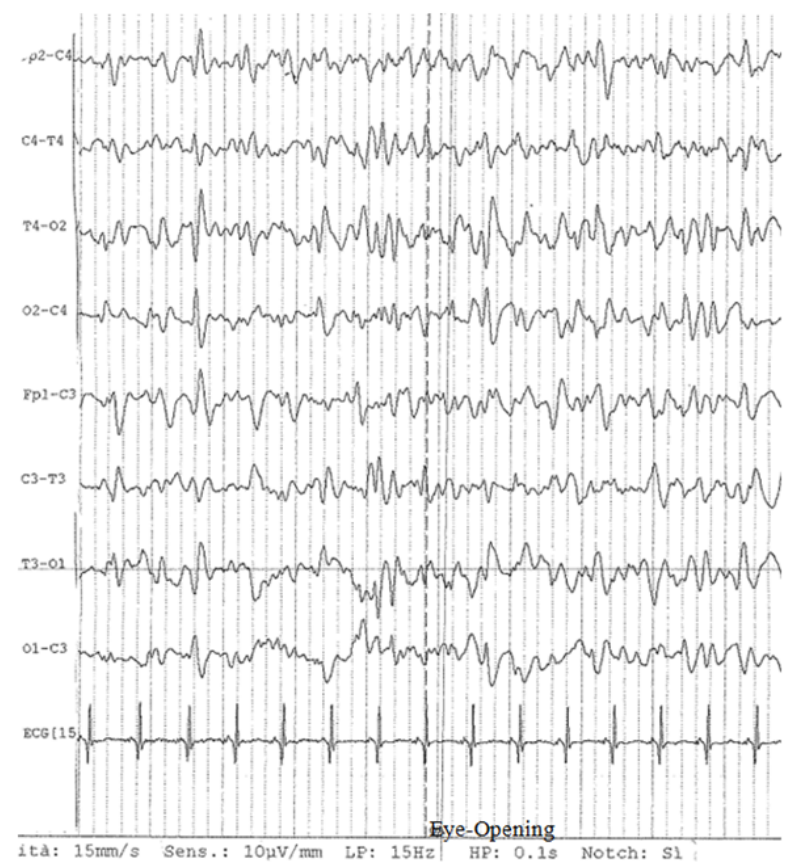

1 (A)

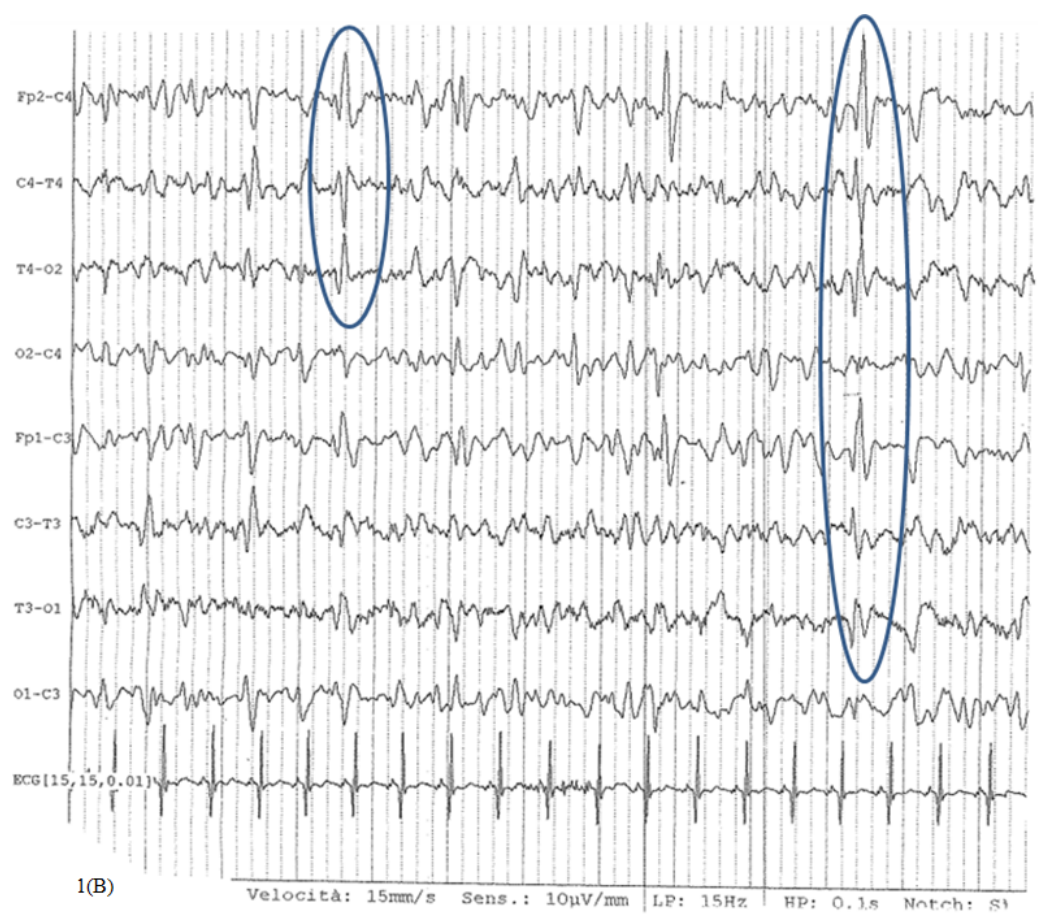

Figure 1. EEG during acute encephalopathy: slowing tracing characterized by theta and delta waves A: trace not responsive to eye-opening, B: anomalies on frontal-temporal-central regions of right side with contra-lateral diffusion

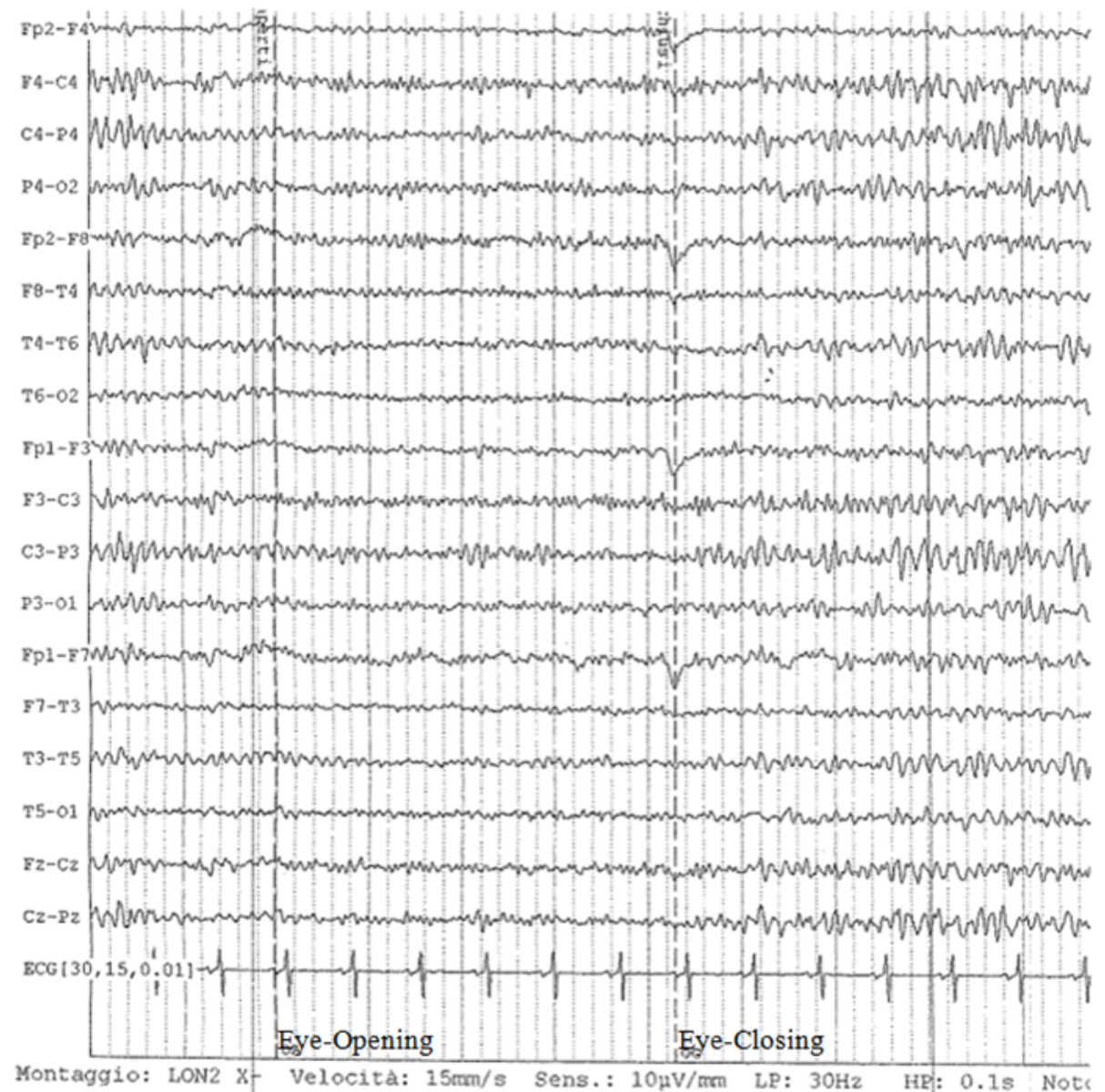

Figure 2. EEG of control (post-therapy with thiamine): restoration of alpha-rhythm characterized by normal response to eye-opening (stop signal reaction) and development of beta waves. 


\section{Conflict of interest}

The authors doesn't have any conflict of interest on the content presented in this case-report.

\section{Funding}

The development of this work was supported by Associazione Onlus ARTIM (Associazione per la Ricerca e le Terapie Innovative per le Mielolesioni).

\section{References}

1. Frankenfield D (2006) Energy expenditure and protein requirements after traumatic injury. Nutr Clin Pract 21: 430-437. [Crossref]

2. Laforenza U, Patrini C, Gastaldi G, Rindi G (1990) Effects of acute and chronic ethano administration on thiamine metabolizing enzymes in some brain areas and in other organs of the rat. Alcohol Alcohol 25: 591-603. [Crossref]

3. Singleton CK, Martin PR (2001) Molecular mechanisms of thiamine utilization. Curr Mol Med 1: 197-207. [Crossref]

4. Todd KG, Hazell AS, Butterworth RF (1999) Alcohol-thiamine interactions: an update on the pathogenesis of Wernicke encephalopathy. Addict Biol 4: 261-272. [Crossref]

5. Farr SA, Scherrer JF, Banks WA, Flood JF, Morley JE (2005) Chronic ethanol consumption impairs learning and memory after cessation of ethanol. Alcohol Clin Exp Res 29: 971-982. [Crossref]

6. Van Dijck A, Van Daele W, De Deyn PP (2012) Uremic Encephalopathy, Miscellanea on Encephalopathies - A Second Look, Dr. Radu Tanasescu (Ed.), ISBN: 978-953 51-0558-9
7. Dreyfus pm (1965) the regional distribution of transketolase in the normal and the thiamine deficient nervous system. J Neuropathol Exp Neurol 24: 119-129. [Crossref]

8. Sterzel RB, Semar M, Lonergan ET, Treser G, Lange K (1971) Relationship of nervous tissue transketolase to the neuropathy in chronic uremia. J Clin Invest 50: 2295-2304. [Crossref]

9. Young, GB, DeRubeis DA. Metabolic encephalopathies. In: Young, GB, Ropper, AH, Bolton, CF, (Eds), Coma and Impaired Consciousness, McGraw-Hill, 1998. p.307

10. Kirshblum SC, Burns SP, Biering-Sorensen F, Donovan W, Graves DE, et al. (2011) International standards for neurological classification of spinal cord injury (revised 2011). J Spinal Cord Med 34: 535-546. [Crossref]

11. Jung YC, Chanraud S, Sullivan EV (2012) Neuroimaging of Wernicke's encephalopathy and Korsakoff's syndrome. Neuropsychol Rev 22: 170-180. [Crossref]

12. Park SH, Kim M, Na DL, Jeon BS (2001) Magnetic resonance reflects the pathological evolution of Wernicke encephalopathy. J Neuroimaging 11: 406-411. [Crossref]

13. Weidauer S, Nichtweiss M, Lanfermann H, Zanella FE (2003) Wernicke encephalopathy: MR findings and clinical presentation. Eur Radiol 13: 1001-1009. [Crossref]

14. Dionyssiotis Y (2012) Malnutrition in spinal cord injury: more than nutritional deficiency. J Clin Med Res 4: 227-236. [Crossref]

15. Walters JL, Buchholz AC, Martin Ginis KA; SHAPE-SCI Research Group (2009) Evidence of dietary inadequacy in adults with chronic spinal cord injury. Spinal Cord 47: 318-322. [Crossref]

16. Moradi H, Said HM (2016) Functional thiamine deficiency in end-stage renal disease: malnutrition despite ample nutrients. Kidney Int 90: 252-254. [Crossref]

Copyright: $(2018$ Stampacchia G. This is an open-access article distributed under the terms of the Creative Commons Attribution License, which permits unrestricted use, distribution, and reproduction in any medium, provided the original author and source are credited. 